\title{
Numerical modelling of the effect of wettability, interfacial tension and temperature on oil recovery at pore-scale level.
}

\author{
IYI, D., BALOGUN, Y., OYENEYIN, B. and FAISAL, N.
}




\title{
Numerical Modelling of the Effect of Wettability, Interfacial Tension and Temperature on Oil Recovery at Pore-Scale level
}

\author{
Draco Iyi, Yakubu Balogun, Babs Oyeneyin, Nadimul Faisal
}

\begin{abstract}
A numerical investigation into the effect of wettability and temperature on oil recovery with a hot water injection at different temperatures is reported in this paper. The computational domain is a two-dimensional porous medium (reservoir) maintained at a fixed temperature with pore spaces of varying sizes and interconnected pore-throats. ANSYS-Fluent VOF (volume of fluid) model was used to simulate the two-phase transport through the reservoir with hot water injections at varying temperatures (20, 40 and 60 ${ }^{\circ} \mathrm{C}$ ) and wettability contact angles of $45^{\circ}, 90^{\circ}$ and $150^{\circ}$. In addition, an investigation was conducted on the effect of combined interfacial tension and matrix wettability on oil recovery process at low and high interfacial tension of $0.025 \mathrm{~N} / \mathrm{m}$ and $0.045 \mathrm{~N} / \mathrm{m}$ respectively for the three different wettability contact angles.

The results showed that, the displacement behaviour of water and oil-wet system is affected significantly by the contact angle with a profound effect on the oil recovery factor. In the water-wet case (with the water wetting the matrix wall and the oil phase surrounded by water), relatively more oil is displaced from the domain thereby improving the oil recovery factor. The water-wetter system resulted in about $35-45 \%$ oil recovery than the oil-wet system, with the unrecovered oil mainly adhering to the wall region of the pore bodies for oil-wet system. For the intermediate wet case, initial fluid distribution is seen to have a more significant effect on the displacement behaviour than the contact angles. In conclusion, by altering the wettability from oil-wet to water-wet condition, the oil recovery rate is improved. The results from this study are consistent with the experimental and numerical studies in literature and it will further enhance the understanding of the phenomenon that is critical to the mechanism of recovery such as surfactant and polymer flooding process.
\end{abstract}

Keyword: oil recovery, wettability, interfacial tension, temperature, contact angle, Computational Fluid Dynamics. 


\section{Introduction}

A mix of interacting factors and rock/fluid properties from the pore scale up to the macro and field scale affects the recovery of oil from reservoirs. The need for a comprehensive understanding and characterisation of the various interacting factors is essential for optimum recovery and an enhanced efficiency and cost effectiveness of petroleum resources recovery. The characterisation of these operating factors was been the subject of intense research over the last decades [1]. One of the key fundamental pore-scale properties that controls the distribution and displacement of fluid in the reservoir is wettability. The wettability of petroleum reservoirs is the most significant factor that control the oil recovery rate and results in a profound effect on petroleum production. It is thus vital to investigate the wettability conditions of the reservoir as it directly affects the multiphase flow characteristic, which influences the different recovery strategies. Consequent to a comprehensive literature review of reservoir wettability conditions and Pore-scale network model, the work reported in this paper focus on the effect of temperature on a wettability contact angle and combined interfacial tension with matrix wettability on oil recovery process.

\section{Literature review}

\subsection{Wettability conditions of reservoir}

Wettability is the spreading and adherence affinity of one fluid to a solid surface in the presence of other immiscible fluids measured by the contact angle as shown in Figure 1 [2]. Thus, in a water-wet media, water preferentially adheres to the surface of the grain solid (hydrophilic) while that of oil-wet media have the oil phase adhering to the solid surface (hydrophobic). The various categories of formation wettability include (a) strongwet, (b) weak-wet, (c) intermediate-wet or neutral-wet, (d) fractional-wet, (e) mixed-wet. When a formation is strongly wetted or weakly wetted, one of the immiscible fluids adheres to the solid surface, while in intermediate or neutral-wettability conditions, this preferential adherence property of the surface is absent and thus both immiscible fluids present have almost equal affinity to the solid surface. Fractional wetting on the other hand, refers to a condition of spatial variation in the wetness of solid surfaces, while mixed wettability is a type of fractional wettability as the walls of the pore space and pore throats have an affinity to different fluids $[1,3]$. In natural porous media, different factors affect the wettability conditions ranging from the surface roughness, immobile adsorbed liquid layers, as well as the adsorption properties of the constituting minerals $[4,5,6]$. It has been reported that the most common formations with minerals such as quartz, carbonates, and sulphates are strongly water wet [5].

A fundamental assumption about the petroleum reservoir is that it is always strongly water-wet. This assumption is because the reservoir was originally a water bearing aquifer prior to the migration of oil from the source rock through migratory pathways to displace some contained water and fill the reservoir now containing both oil and water. Chinedu et al., [6] stated that water is contained in the pore volumes of the reservoir with the migrated oil and the final wettability determination is dependent on the constituents of the oil. The final wettability is affected by whether the oil contains polar compounds and high molecular paraffin. In additions, it is also affected by the distribution of minerals, reservoir rock type as well as the salinity of the connate water. This finding was further buttressed by Blunt et al., [7] stating that petroleum reservoirs are strongly water-wet as oil-wetness characteristics are observed in many soils contaminated by oil. Buckley et al., [8] stated the reason for this alteration to be the continued contact of the oil phase with the solid surface results in the adherence of the surface-active components of the oil to the solid surface thereby changing the surface wettability. 


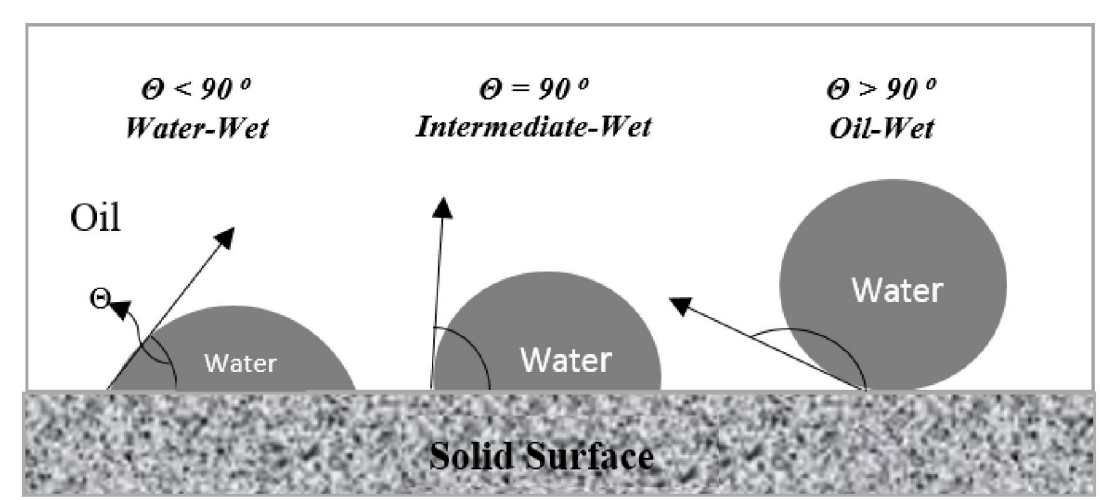

Figure 1: Different wettability contact angles.

The oil-water wetting preference of the formation affects many facets of reservoir performance, mainly in water flooding and enhanced oil recovery methods. Therefore, wrongly assuming a water-wet reservoir condition could lead to irreversible reservoir damage [9]. Therefore, the understanding of the wettability condition of oilbearing formation is vital for optimizing oil recovery. The demand for crude oil is continuously increasing due to industrial development and an increase in world population. The increase in global demand has necessitated improvements in the recovery strategy with water flooding which is one of the least expensive and easily implemented techniques. With the reservoir been a multiphase flow domain, understanding wettability becomes important to optimise recovery [10]. The original wettability of a formation and altered wettability during and after hydrocarbon migration influence the profile of initial water saturation and production characteristics in the formation [9]. Further information on wettability and its corresponding effect on the exploitation petroleum resources can be found in literature $[2,6,7,11]$.

Olugbenga and Manuel [5] experiment investigated the effects of wettability on capillary pressure, relative permeability, and irreducible saturation using a porous plate. The study reported how the wettability alteration of a medium of water to oil-wetness affect the multiphase flow properties. The initial water-wet samples with porosities ranging from $23 \%$ to $33 \%$ and absolute air permeability of 50 to $233 \mathrm{mD}$, yielded an irreducible wetting phase saturation of $19 \%$ to $21 \%$ when tested as water wet samples under airbrine system. In addition, they altered the wettability to oil-wet using a surfactant with the test yielding a wetting phase (oil) irreducible saturation of $25 \%$ to $34 \%$ and concluded that a change of the wettability from water-wet to oil-wet results is an improvement of the wetting phase (oil) recovery.

Zhang and Austad [12], investigated the effect of temperature and ionic contents on wettability and oil recovery from carbonate rocks. They conducted a series of experiments by spontaneous imbibition of water with different sulphate concentrations into a homogenous chalk core of permeability between 2-5 $\mathrm{mD}$ at different operating temperatures. Their results showed significant improvement of the oil recovery with increasing sulphate concentration in the injection fluid for a moderate water wet and preferential oil wet chalk samples. In addition, a better efficiency in the wettability alteration process in presence of sulphate with increase in temperature was reported. Another study on carbonate reservoirs was carried out by Kallel et al., [13] on the effect of wettability distributions on oil recovery from microporous carbonate reservoirs. They used a qualitatively wettability alteration scenario to implement a two-phase flow network model to capture a diversity of pore shapes. Their results revealed that wettability effects are considerably significant in the carbonate network due to the micro-pores effects on oil recovery.

\subsection{Pore-scale network model}

Blunt et al., [14] investigated the effect of wettability on water-flood oil recovery with a pore-scale network model approach. They explored several multiphase flow 
phenomena using pore networks extracted from unconsolidated sand pack, a poorly consolidated sandstone cores, a granular carbonate and Berea sandstone. Their results showed that in a uniformly wet system, less water-wetness increases recovery and reaches a maximum for oil-wet condition in which the recovery becomes relatively constant at contact angle about $100^{\circ}$. In addition, their results also showed that the oil-wet fraction affects the recovery in a mixed-wet media more than the contact angle in the oil-wet regions with optimal recovery occurring when a small fraction of the system is water-wet. Mohammadmoradi and Apostolos [15] implemented a direct quasi-static simulation approach to investigate the effect of wettability on water-flood performance in partially saturated microstructures. Their results showed that the electrical and hydraulic conductivity influence the wettability that is significance in shaping the fluid pathways and two phase spatial distribution in the formation. Their findings are in agreement with previous studies that wettability is a critical factor controlling fluid distribution in a porous medium, but disagrees with the reports of oil-wetted favouring recovery. Their findings also showed that oil-wetness speeds up water breakthrough time and decreases oil recovery during spontaneous imbibition.

Mingming and Wang [16] used a two-dimensional simplification of a porous media pore volume geometry to study the process of hot water flooding in a water/oil twophase flow for enhanced oil recovery. They adopted the volume of fluid (VOF) multiphase flow model to capture the position of the multiphase fluid (oil/water) interface and heat transfer physics. The behaviours of hot water flooding at pore-scale under different wettability condition were investigated and they reported a significant effect of the contact angle on the original oil saturation and the displacement process for the oil-wet media and showed an increase in the oil recovery with increasing temperature. Zhao and Wen [17] employed an idealised geometry similar to that of Mingming and Wang [16] to investigate the effect of wettability and interfacial tension on flooding process for enhanced oil recovery. Their findings showed that wettability effect on recovery at pore scale in oilsaturated pores is significant in a water-wet scenario. They concluded that a good mixture of both water wettability alteration and a low capillary effect could potentially result to an ideal EOR result.

A thermal recovery method involving injection of steam or hot water is known to change the wettability of reservoir rocks [1]. Temperature effect on wettability has been shown to improve or change the hydrophilic nature of the reservoir rock [18]. Dangerfield and Brown [19] change original hydrophilic rock to hydrophobic via oil deposit on the surface of the rock due to the adsorption of ionic compounds of crude oil. Schembre et al., [20] investigated the effect of water imbibition at elevated temperatures on wettability alteration and oil recovery. They conducted experiments at temperatures from 45 to 230 ${ }^{\circ} \mathrm{C}$ using nine different reservoir core samples of permeability ranging from 0.2 to $0.7 \mathrm{mD}$ and porosity of 45 to $65 \%$. Their results showed that the increase in temperature lead to a significant increment in imbibition rate and oil recovery, with a shift in the wettability index from intermediate and weakly water-wet to strongly water-wet.

Several research works on the effect of wettability on the macroscopic behaviour of fluids has been reported in literature. Nevertheless, there is no definite consensus on the optimum wettability condition for the most favourable oil recovery performance as some of the studies reveal highest recovery under oil-wet conditions [5, 14], while others asserts that recovery efficiency is achieved under water-wet conditions $[15,16]$. In addition, the application of imaging tools has been used to analyse the pore-scale phenomenon and its influence on the immiscible displacement process, but with a major challenge as it is rather difficult to control and vary the wettability of the porous matrix experimentally.

In this study, a Computational Fluid Dynamics (CFD) method is used to simulate two-phase flows at the pore level. A major advantage of direct simulations, as CFD is the possibility of obtaining a realistic representation of the digital porous system with models that captures the intricate physics present in pore-scale flows. The main objective of this study is to delineate the pore-scale behaviour of immiscible displacement under varying wettability and interfacial tension conditions. The work reported in this paper provides a 
detailed representation of the interfacial phenomena by varying the wetness conditions of porous media, interfacial tension and fluid viscosity.

\section{Numerical model description}

A multiphase flow simulation at pore scale and capturing the interface between the immiscible fluids is usually a matter of interest. A numerical modelling of a twodimensional idealised poly-disperse porous media (Figure 2) was simulated with ANSYS Fluent ${ }^{\circledR} 18.1$ to simulate the effects of varying porous media wetness conditions, interfacial tension and fluid viscosity on oil recovery. The Volume of Fluid (VOF) method was used to simulate a water-flood operation in a series of interconnected pore spaces with the incorporation of interface tracking model to track the oil/water interface. Three different injection temperature scenarios $\left(20,40\right.$ and $60^{\circ} \mathrm{C}$ ) under varying wettability conditions for the matrix wall were simulated. Water at different temperature $(20,40$ and $60{ }^{\circ} \mathrm{C}$ ) was injected into a high temperature $\left(80^{\circ} \mathrm{C}\right)$ porous domain of $80 \%$ oil saturation and an irreducible water saturation of $20 \%$. Two different interfacial tension values of $0.025 \mathrm{~N} / \mathrm{m}$ and $0.045 \mathrm{~N} / \mathrm{m}$ was used each to model the different contact angle conditions of water-wet $\left(45^{\circ}\right)$, intermediate-wet $\left(90^{\circ}\right)$ and oil-wet $\left(150^{\circ}\right)$. To simulate the interfacial tension and wettability conditions of the system; the phase interaction physics capability in ANSYS Fluent ${ }^{\circledR}$ software was used. In the phase interaction panel, the interfacial tension was set and the wettability conditions imposed by the wall adhesion option after the specific contact angle was specified under the boundary conditions settings for the grain walls.

\subsection{Model geometry}

The two-dimensional pore scale geometry used in this study is a $22 \mathrm{~mm}$ by $10 \mathrm{~mm}$ rectangle block shown in Figure 2. The micromodel in this study is made of polydisperse solid grains having two different diameters of 1 and $2 \mathrm{~mm}$ which is representative of commercial grade silica grade silica sand 8/16 [21]. Pore-bodies and pore throats of varying dimensions characterize the model. While some study utilises a homogenous porous medium with similar diameter for all the grains, it is obvious that that is not a realistic representation of a natural porous media. The different diameter sizes for the sand grains has been used to mimic the true nature of a natural porous system with varying grains sizes and thus heterogeneous flow characteristics. The pore throat width was varied between $0.10 \mathrm{~mm}$ and $0.35 \mathrm{~mm}$ to mimic a low and high permeability zone as representative of a natural porous media.

The pore volume of the sample is $1.28 \times 10^{-4} \mathrm{~m}^{3}$, which gives a porosity of approximately $58 \%$. Single-phase flow simulation with water indicates an absolute permeability of $8.6 \times 10^{-9} \mathrm{~m}^{2}$. At the inlet and outlet face, a gap of approximately $1 \mathrm{~mm}$ was allowed before the first set of grains. Although a natural rock 2D slide would have a spatially periodic matrix at these faces, the gaps imposed here would allow the flow to develop before meeting the first sets of obstructions in the solid grain matrix. While an idealised geometry as this do not reflect the 3D connectivity of real porous media, they can be adopted as computationally affordable alternatives to 3D pore-scale models that allows for more detailed visualization of the intricate physics in a much clearer way than the 3-D models. Another advantage for the application of micromodels is the prospect of designing, fabricating and studying different shapes and patterns.

A no-slip boundary condition was imposed on the grain walls and on all the lateral sides. The flow domain is initially saturated with phase- 1 (oil) at $80 \%$ and phase-2 (water) at $20 \%$ and water was injected at a constant velocity of $0.005 \mathrm{~m} / \mathrm{s}$ from the inlet and 0 Pa pressure was specified as the outlet boundary condition. A velocity inlet condition has been used in the model as a standard practice in situations where the injection flow rate or velocity is known without information of the pressure at the inlet. As obtained in laboratory core flood experiments, an injection flow rate is imposed at the inlet and the pressure drop is recorded with installed transducers, in this set of simulations, the 
numerical model computes the pressure at the inlet from the imposed velocity condition and other flow parameters [22]. Based upon the injection velocity, pore diameters and velocity of fluids the flow was assumed laminar and as such, no turbulence model considered.

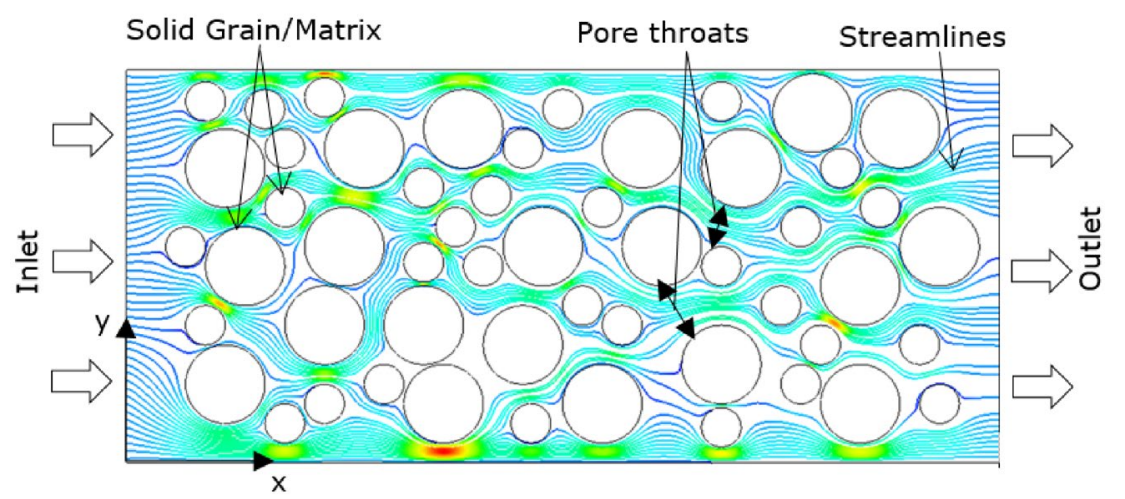

Figure 2: the 2D pore-scale media configuration.

For a better capturing of the occurrences around the walls and for the accuracy of the results, the grid around the individual grain wall region was structured and refined with fine meshes. A number of sensitivity studies were carried out to determine the optimum inflation layers, mesh sizes and number of control volumes needed to ensure that the computed profiles of the oil/water interface are grid independent. The final mesh used for the simulations has a total control volume of 39817 (Figure 3 ). The time steps sizes used for the simulation is 0.0005 and PISO scheme for the pressure-velocity coupling, PRESTO for the pressure discretization and Geo-Reconstruct for the volume fraction.

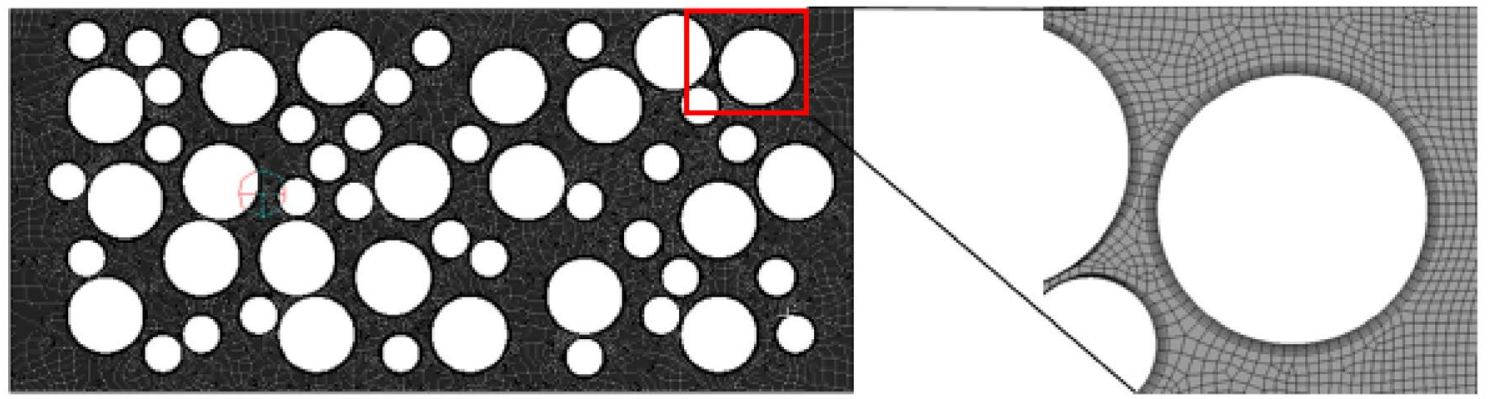

Figure 3: Grid used for the study showing the refined inflation layers.

\subsection{Properties of fluid and the porous media}

All the simulations were conducted with constant fluid property except for the viscosity of the oil phase owing to the heat transfer between the fluids and solid matrix walls. The water phase has a viscosity of $0.001 \mathrm{~kg} / \mathrm{m}-\mathrm{s}$ while the oil has a varying viscosity with respect to operating temperature (Figure 4 ). While the oil phase was simulated with a temperature dependent viscosity, the water phase viscosity was kept constant as experimental observation on the variation of water viscosity with temperature is minimal and as such has a negligible effect in flooding a highly viscous oil phase. We conducted a preliminary experiment to obtain the viscosity data of motor oil at different temperatures using a fann model 35 viscometer. The viscosity ratio $\left(\mu_{w} / \mu_{o}\right)$ for the simulations was found to be between $0.007-0.081$ which is less than $1 \times 10^{3}$ as recommended by ANSYS Fluent ${ }^{\circledR}$ to avoid convergence difficulties. The density of the displacing fluid (water) is 1000 $\mathrm{kg} / \mathrm{m}^{3}$ while that of oil is $865.8 \mathrm{~kg} / \mathrm{m}^{3}$. Although, it was expected that the density of oil will be affected by various factors such as temperature and pressure, however, only the influence of temperature on viscosity was considered in this study. In addition, the effect of temperature on the fluid thermal conductivity and specific heat capacity was not 
considered. A survey of the literature showed a near linear decrease in the oil/water interfacial tension, $\sigma_{\text {ow }}$ with increase in temperature $[23,24,25]$, and a $1^{\circ} \mathrm{C}$ increase in temperature resulted in a $0.05 \mathrm{mN} / \mathrm{m}$ decrease in oil/water interfacial tension using reference values of $47 \mathrm{mN} / \mathrm{m}$ at temperature of $60^{\circ} \mathrm{C}$ [16].

The viscosity of the primary phase (oil) as a function of local temperature was incorporated through a User-Defined Function (UDF). The experimental viscosity data were used to determine a function for corresponding oil phase viscosity for temperature ranging from $20^{\circ} \mathrm{C}$ to $100^{\circ} \mathrm{C}$. The model for the viscosity with corresponding temperature is given in Eq. (1). As seen in the function below, at temperatures above $100{ }^{\circ} \mathrm{C}$, the oil viscosity is $12.3 \mathrm{CP}$, when the temperate is below $20^{\circ} \mathrm{C}$ the oil viscosity is $142 \mathrm{cP}$ while for temperatures between the ranges of 20 to $100{ }^{\circ} \mathrm{C}$, the function is used to calculate the viscosity of the oil phase.

$$
\mu=f(T)=\left\{\begin{aligned}
0.0123(\mathrm{~kg} / \mathrm{ms}), & T>373 K \\
4504.7 e^{-0.035 T}(\mathrm{~kg} / \mathrm{ms}), & 293 \mathrm{k} \leq T \leq 373 K \\
0.142(\mathrm{~kg} / \mathrm{ms}), & T<293 \mathrm{~K}
\end{aligned}\right.
$$

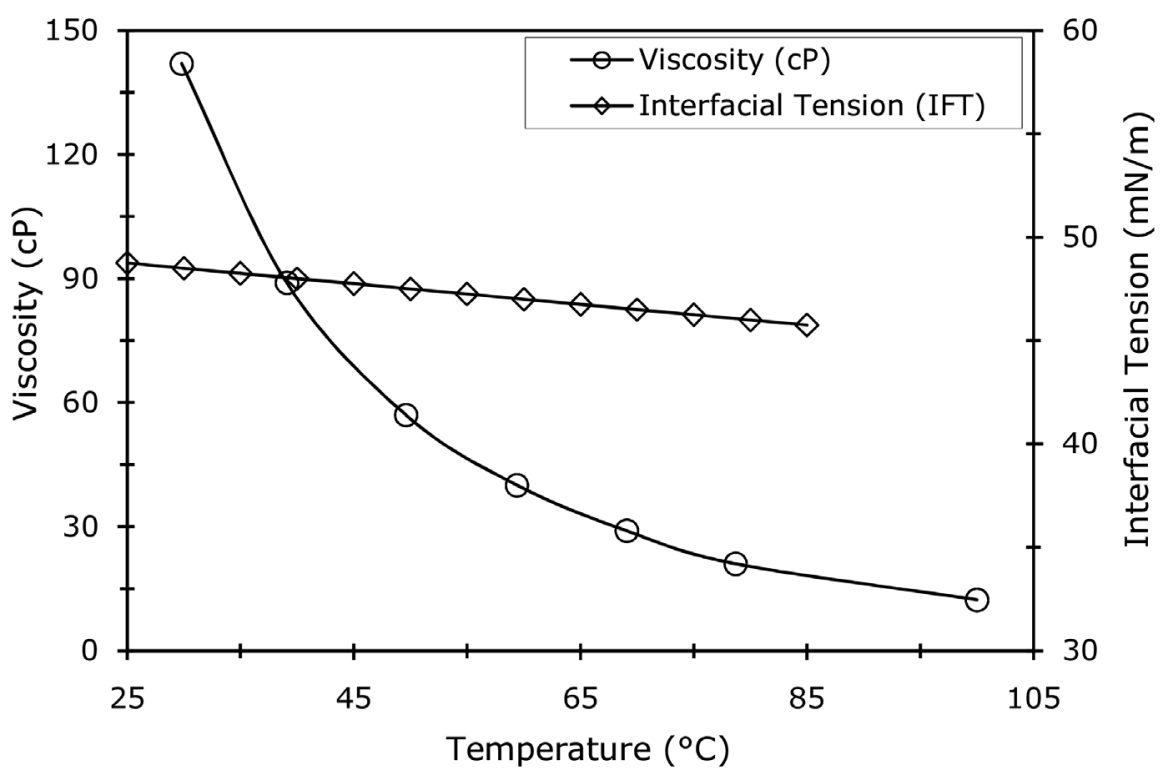

Figure 4: Variation of oil viscosity with temperature.

\subsection{Volume-Of-Fluid (VOF) method}

The VOF method is a numerical modelling technique for tracking and detecting the free surface or fluid-fluid interface and it's model two or more immiscible fluids by solving a single set of momentum equations and tracking the volume fraction of each of the fluids throughout the domain [24]. The model formulation works on the basis that the two or more fluid phases are immiscible and non-interpenetrating and within each control volume, the volume fractions of all the fluid phases is equal to one. A volume averaged value variables and property is assigned to each of the phases, if the volume fraction of each phase is known at each location. Therefore, the variables and properties within a given cell is a purely representative either of a given phase, or of results from a mixture of the phases. Typically, the volume fraction of oil phase $\left(a_{0}\right)$ equals to one if the cell is completely occupied by oil $\left(a_{o}=1\right)$, while it equals to zero if the cell is completely occupied by water $\left(a_{o}=0\right)$. If the cell contains the oil-water interface, then the volume fractions of oil and water lies between $0-1 \quad\left(0<a_{0}<1\right)$. The governing equations solved in the VOF models include the standard mass, momentum and energy conservation equations with the inclusion of the volume fraction equation as presented in Eq. (2-6). 
$\frac{\partial(\rho \vec{u})}{\partial t}+\nabla \cdot(\rho \vec{u} \vec{u})=-\nabla p=\nabla \cdot\left[\mu\left(\nabla \vec{u}+\nabla \vec{u}^{T}\right)\right]+\vec{F}$

$\frac{\partial(\rho T)}{\partial t}+\nabla \cdot(\rho \vec{u} T)=\nabla \cdot\left(\frac{k}{c_{p}} \nabla T\right)$

$$
\frac{\partial \alpha_{w}}{\partial t}+\vec{u} \cdot \nabla \alpha_{w}=0
$$

Where $\vec{u}=(u, v)$ is the velocity vector, $\rho$ is the volume-averaged density and $p$ is the pressure. The coefficient of kinetic viscosity is $\mu$, the surface tension force per unit volume is $\vec{F}, T$ is the temperature, $k$ is the thermal conductivity and $c_{p}$ is the specific heat capacity.

The fluid properties computed in the transport equations are determined by the presence or absence of the component fluids in each computational cell. When a computational cell or control volume is completely filled by a single phase, only the properties of the phase are used in the equations. However, when the fluid interface is within the control volume, the mixture properties of the two phases are used to compute the volume fraction weighted average. The following equations were used for computing the properties in a control volume. In a two-phase oil-water system for example, denoting the oil and water by the subscripts $o$ and $w$ respectively, and if the volume fraction of the water is being tracked, the density in each cell is given in Eq. (6). Other fluid properties [e.g., viscosity (Eq. (7)] were computed in a similar manner.

$$
\begin{aligned}
& \rho=\alpha_{w} \rho_{w}+\left(1-\alpha_{o}\right) \rho_{o} \\
& \mu=\alpha_{w} \mu_{w}+\left(1-\alpha_{o}\right) \mu_{o}
\end{aligned}
$$

\subsection{Surface tension, wall adhesion and capillary number}

Surface tension plays a dominant role in a two-phase immiscible fluid flow at microscale level. The continuum surface force (CSF) model for surface tension by Brackbill et al., [26] was implemented in ANSYS Fluent ${ }^{\circledR}$ [27] model through the source term in the momentum equation. Equation (8) was used to approximate the surface tension force per unit volume $\vec{F}$ in the momentum equation

$$
\vec{F}=\sigma \frac{2 \rho k_{w} \nabla \alpha_{w}}{\left(\rho_{w}+\rho_{o}\right)}
$$

Where, $\sigma$ is the surface tension coefficient and $\mathrm{k}$ is the interface curvature computed from $k=-(\nabla \cdot \hat{n})$. The unit normal vector of the interface is represented as $\hat{n}$. The contact angle with the wall was used to adjust the unit normal vector of the interface $(\hat{n})$ in the cells near the wall. The surface normal to the live cell next to the wall as $\hat{n}=$ $\hat{n}_{w} \cos \theta_{w}+\hat{t}_{w} \sin \theta_{w}$. Where $\hat{n}_{w}$ and $\hat{t}_{w}$ are the unit vectors normal and tangential to the wall, respectively.

The capillary number is a dimensionless quantity that characterizes the ratio of viscous forces to the interfacial tension forces acting across the fluid-fluid interface denoted by $\mathrm{Ca}$ [Eq. (9)]. For a flowing liquid, if $\mathrm{Ca}>1$, then viscous forces is more dominant relative to the interfacial forces. However, for $\mathrm{Ca}<1$, the interfacial forces dominates the flow and viscous forces are negligible. The capillary number is usually large for high-speed flows compared to low-speed flows. A typically capillary flow through pores in the porous reservoir have $\mathrm{Ca}$ of about $10^{-6}$, and flow in drill pipes have $\mathrm{Ca}$ of about 1 [28]. 


$$
C a=\frac{\mu V}{\sigma}
$$

\subsection{Validation of the numerical methodology}

A preliminary study was conducted to validate the numerical methodology against a recovery factor data adapted from a core flood experiment of Ahmadi et al. 2016 [22]. The design and operating conditions of the numerical study are similar to the experimental conditions with the injection temperature of $20^{\circ} \mathrm{C}$ and inlet velocity of $2.94 \times 10^{6} \mathrm{~m} / \mathrm{s}$. The recovery factor results at different time intervals were plotted against the core flooding experimental data as shown in Figure 5. The results displayed a favourable comparison between the numerical and experimental data with less than $\pm 2.5 \%$ error margin.

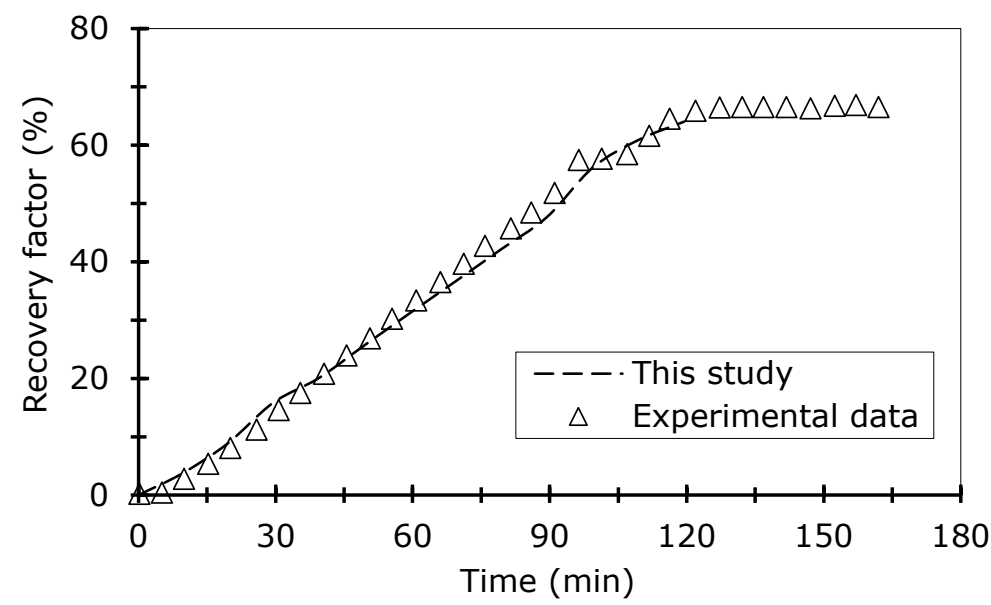

Figure 5: comparison of the recovery factor plots between experiment and numerical data.

\section{Results and discussion}

The results of the hot water-flooding processes involving the different wettability and interfacial tension scenarios have been presented and analysed in this section. The results are in the form of volume recovery factors $(R F)$ which is defined as the volume fraction of oil that was displaced from the porous media and was computed using Eq. (10).

$$
R F=\frac{V_{\text {displaced }}}{V_{\text {initial }}}=\frac{V_{\text {initial }}-V_{\text {residual }}}{V_{\text {initial }}} \times 100
$$

Where $V_{\text {initial }}$ is the initial volume of oil in the domain, $V_{\text {displaced }}$ is the volume of oil displaced, and $V_{\text {residual }}$ is the residual volume of oil left in the domain after the water flooding process.

The respective pressure drop across the computational domain at a different injection temperature under the three wettability conditions considered is shown in Figure 6 . It is observed that the entire wettability scenario shows a reduction in the pressure with an increase in temperature, but become almost insignificant with the water-wet scenario. However, under a low injection temperature, the pressure drops for the intermediate-wet and oil-wet cases shown a higher value in comparison to the water-wet media. This could be attributed to the fact that the oil phase adheres to the solid grain walls and thus resulting in a resistance to flow causing an increased the pressure drop. With the increase in temperature from 20 to $60{ }^{\circ} \mathrm{C}$, it can be observed that the pressure drop reduces and 
almost having the same magnitude with that of the water-wet media. This may be due to the reduction in the oil viscosity and pressure drop with the increase in temperature.

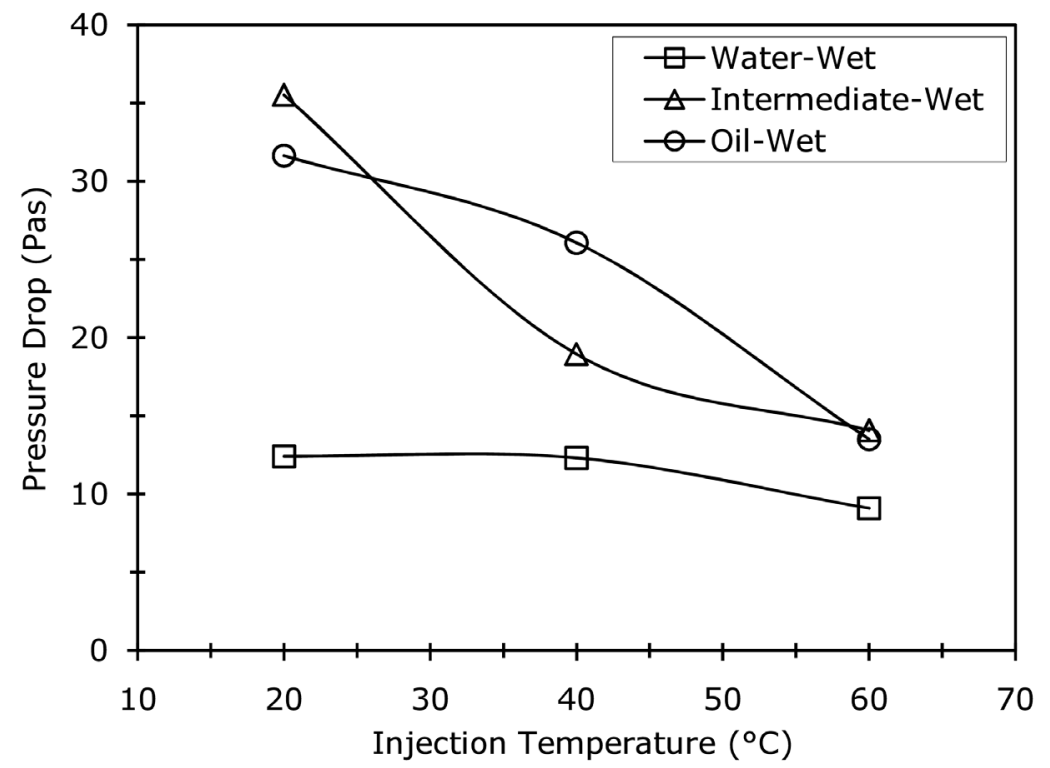

Figure 6: Pressure drops across the domain at different temperature.

\subsection{Combined effect of wettability and temperature on the recovery factor}

The effect of porous media wettability on recovery factor at different injection temperature is presented in Figure 7. The result of the wettability cases presented were conducted using three different water contact angles of $45^{\circ}, 90^{\circ}$ and $150^{\circ}$. As shown in the plot, the recovery factor in the water-wet or hydrophilic (i.e. media with a greater affinity for water than for oil) is highest with values above $70 \%$ and the recovery factor decreases with increasing contact angle with values around $40 \%$ for the intermediate-wet system and less than $20 \%$ of the oil-wet media. Similar results is observed in Figure 8 , where the oil phase sticking to the spherical solid grains for all the oil-wet scenarios. It can be seen that at the transient evolution of the fluid-fluid interface, the water phase convex to the left in the water-wet media with water filling up the small pore spaces while the oil forms globules of varying sizes in the central part of the large pores. The oil phase has no direct contact with the matrix wall, but covered with a thin water film and serving as a form of slippery surface for the oil to be displaced and recovered. In this case, the water breakthrough time is relatively delayed because the displacement process favours the outward flow of the oil phase more than the water.

However, in the case of hydrophobic rock, the reverse of the above process is seen as the cover surface of the rock, creating a form of lubricating lining for the easy passage of the invading water, which results in quicker water breakthrough. In addition, the effect of oil-wetness can also be seen in the pressure profile shown in Figure 6 where at $20{ }^{\circ} \mathrm{C}$, the pressure drop in the water-wet media is about $12 \mathrm{~Pa}$ when compared to the $35 \mathrm{~Pa}$ for the oil-wet media. It is evidence that higher pressure is needed to mobilise the oil from the inner small pores of the media and to detach the oil phase from the walls of the porous media.

The effect of temperature is found to be more predominant in the water-wet media. For the water-wet case and temperature between 20 and $60{ }^{\circ} \mathrm{C}$, a variation of about $17 \%$ is observed in the recovery factor, while almost an insignificant variation is observed in the oil-wet case. It can be explained that water-wetness makes it relatively easier for the oil to be displaced by the invading water while the oil-wet case requires more thermal energy in the system to reduce the oil viscosity; thereby releasing the oil stuck on the matrix wall and displace as much oil as possible. For the low temperature $\left(20^{\circ} \mathrm{C}\right)$ injection 
case, the recovery factor of the water-wet media is seen to be about $72 \%$, while that of the oil-wet media is a little above $10 \%$. However, with increasing temperature, there seems to be a slight reversal in the profile with higher recovery favouring the oil-wet case. This observation is in agreement with the findings of Mingming et al., [16] that reported an increase in the oil recovery with increment in temperature for the oil-wet media. A plausible explanation for this occurrence is that a reduction in the viscosity of a fluid under same interfacial tension results in a reduction in the capillary number, which can be observed in the water-wet displayed in Figure 6. However, an injection temperature of 20 ${ }^{\circ} \mathrm{C}$ leads to a recovery of $72 \%$, and reducing the viscosity of the oil phase by increasing the temperature to $60{ }^{\circ} \mathrm{C}$ and under the same interfacial tension and water-wetness lead to a reduction in the capillary number, which hinders the recovery of the oil, phase $(60 \%)$.

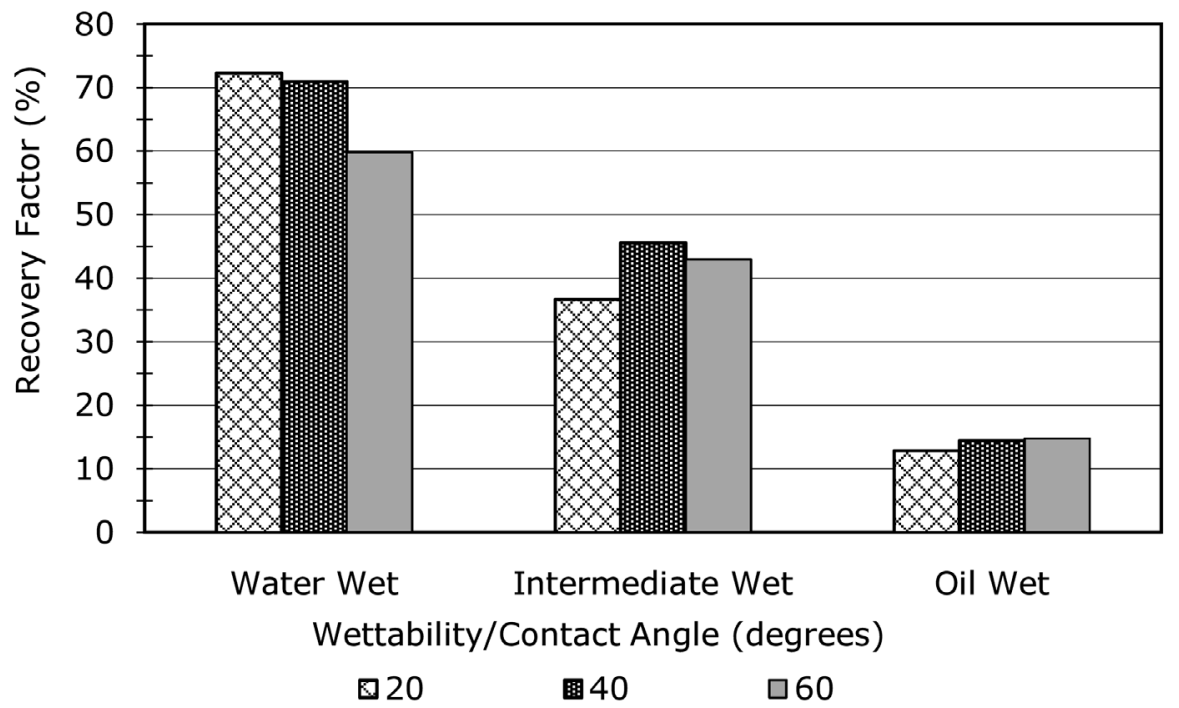

Figure 7: Combined effect of wettability and temperature on recovery factor.

The wettability condition of a reservoir rocks affects the effectiveness of any oil recovery method in use. At the commencement of oil production through primary recovery, the displacement of the oil phase is mainly under the influence of a pressure drop with the oil phase having a relatively high mobility and relative permeability due to its high saturation, making it easy to move in the direction of the wellbore. With the decline in the relative permeability of the oil via the reduction in its saturation (Figure 8 ), water saturation increase by the invading water filling the pore spaces which was earlier occupied by oil and then leaving the remaining oil in the form of isolated globules sandwiched in the water. This make it difficult to extract the oil singularly by the effect of a pressure difference.

With increase in temperature of the injection water (thermal recovery method), more oil is recovered (mainly in the oil-wet media) as shown in Figure 7 and 8 . With the reduction in the oil-viscosity via temperature increase leading to capillary pressure reduction and resulted in the coming together of the oil globules into larger droplets (coalescence). This coalesced oil phase forms a zone or chain-like network of connected oil (oil bank) that easily migrate to the outlet. Besides the reduction in the oil viscosity, other studies have reported that temperature aids in the oil recovery by changing the media wettability in the hydrophilic direction $[1,18,19]$. As reported by Dangerfield and Brown, [19] at high temperatures, ionic compounds separate from the wall of the media resulting in a change of the wettability to become more hydrophilic. Donaldson and Alam [1] reported a similar increase in recovery with an increase in temperature due to the relative permeability increase of oil with increasing temperature. 


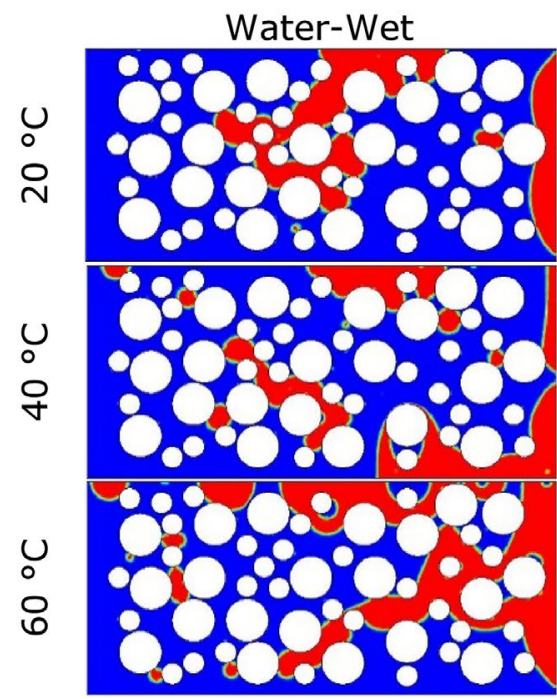

Intermediate-Wet

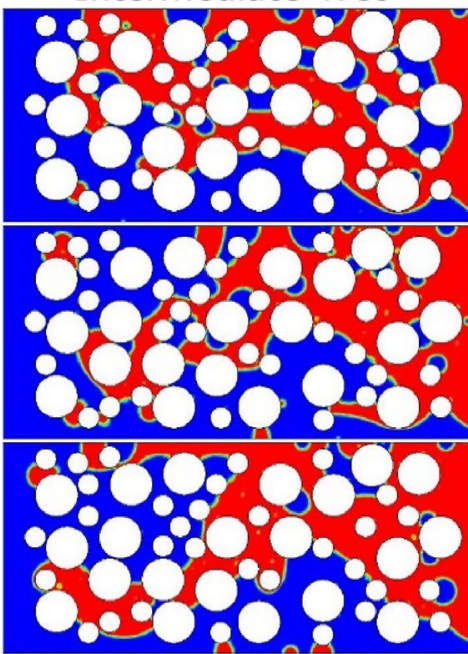

Oil-Wet
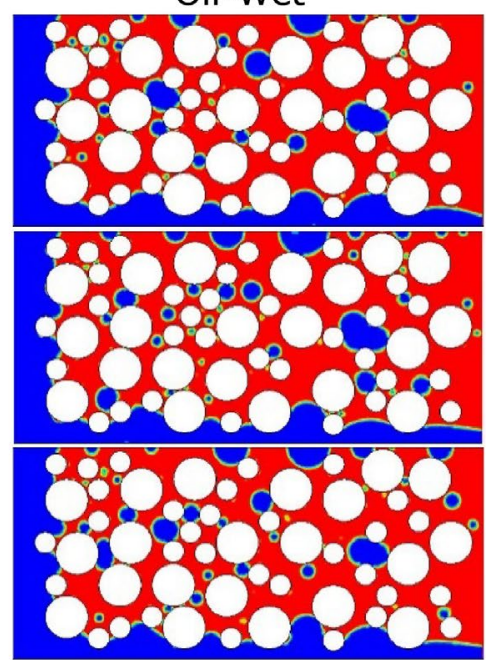

(a) Simulations under low interfacial tension of $0.025 \mathrm{~N} / \mathrm{m}$
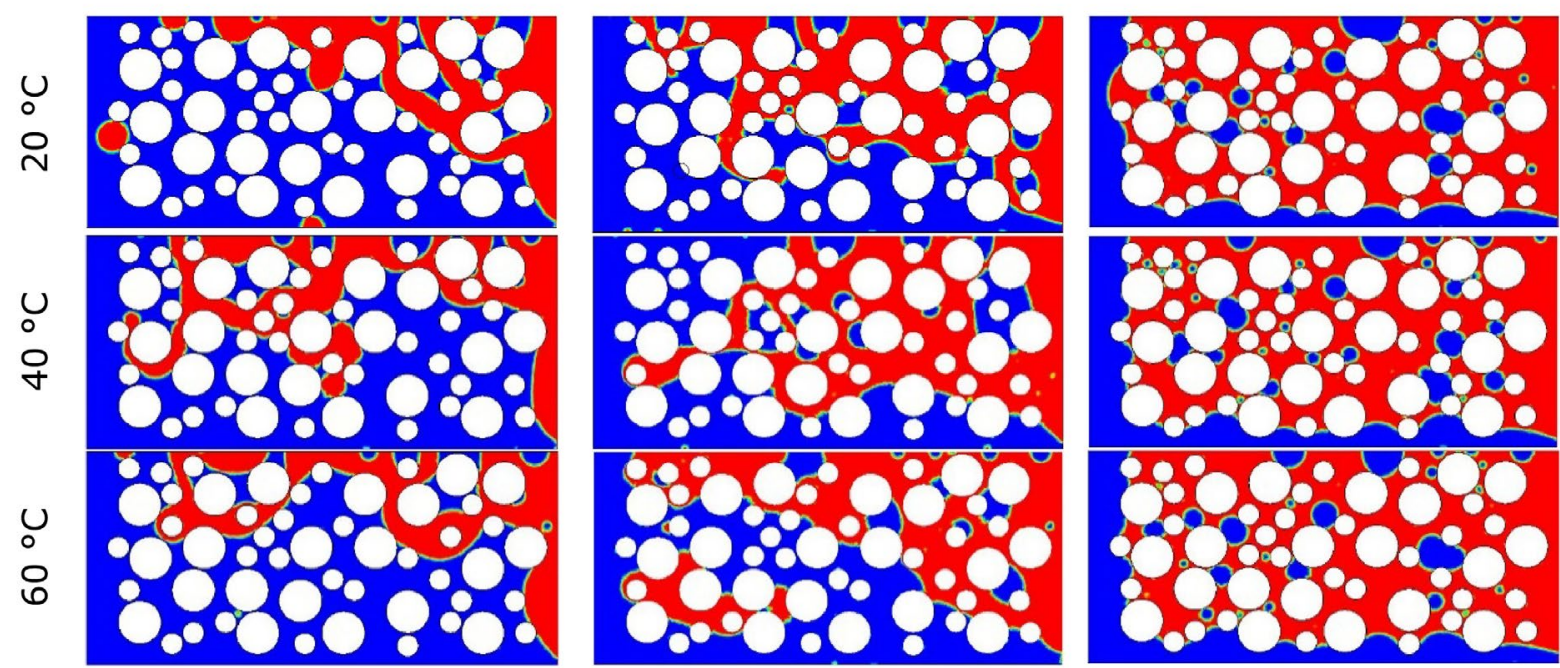

(b) Simulations under high interfacial tension of $0.045 \mathrm{~N} / \mathrm{m}$

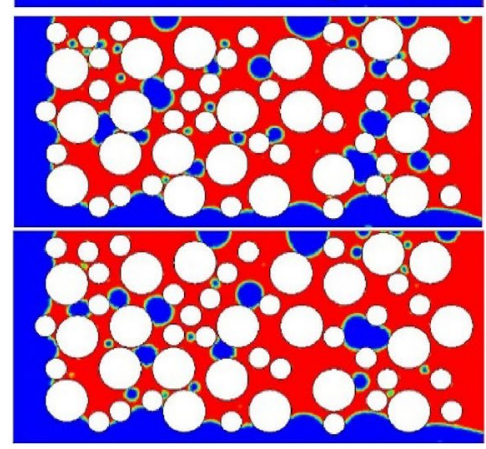

462

463

464

465

Figure 8: Fluid distribution of the different cases (the red and blue colour is oil and water respectively) (a) low IFT and (b) high IFT.

\subsection{Effect of Interfacial Tension (IFT)}

The effect of interfacial tension (IFT) on the displacement process under different wettability conditions are presented the Figure $9(\mathrm{a}-\mathrm{c})$. The relative trend shows that the percentage recovery of oil from the flooding are higher in the cases of lower interfacial tension. The displacement process was simulated under the water-wet $\left(45^{\circ}\right)$, intermediate-wet $\left(90^{\circ}\right)$ and oil-wet $\left(150^{\circ}\right)$ state, at varying injection temperatures. In practice, in the primary oil recovery, approximately $20 \%$ of the original oil in place is recovered depending on the type of reservoir, with a secondary recovery mechanism adding another 15 to $20 \%$ [29]. The quest to recover the left over oil is the aim of every enhanced oil recovery mechanism. As stated by Carcoana [29], the two main factors that determines the recovery of residual oil are the Capillary Number and Mobility Ratio.

It is evident from the results in Figure 9 that a reduction of the interfacial tension leads to a better recovery. This could be explained with the Eq. (9) for the capillary number representing the ratio of viscous to capillary forces. A reduction in the interfacial tension for the same media constriction (pore geometry) resulted in an increase in capillary number which is a significant parameter in oil recovery. In essence, a lower capillary number suggests that capillary forces dominate the flow, while a larger capillary number indicates that the flow is a viscous dominated. In practice, enhanced oil recovery 
mechanism wishes to increase the capillary number in order to reduce trapping. In this regards, Thomas. S, [30] pointed out that capillary number needs to be increased by three orders of magnitude to recover about $50 \%$ of the residual oil saturation.

The benefit of combining IFT and wettability is apparent from this study. For an injection temperature of $20{ }^{\circ} \mathrm{C}$ under high IFT of $0.045 \mathrm{~N} / \mathrm{m}$, a percentage recovery of about $10 \%$ was observed. Reducing only the IFT to $0.025 \mathrm{~N} / \mathrm{m}$ improves the recovery minimally to about $13 \%$. On the other hand, reducing the wettability from the oil-wet of $150^{\circ}$ to intermediate-wet of $90^{\circ}$ results in recovery factor of between $35-45 \%$, and a further reduction of the wettability to water-wet $\left(45^{\circ}\right)$ results in recovery factor of between $60-75 \%$. This clearly shows that, though a low IFT is enough to resist the capillary effects, an improved oil recovery factor cannot be achieved due to the adherence of the oil to the walls caused by the wettability effects.

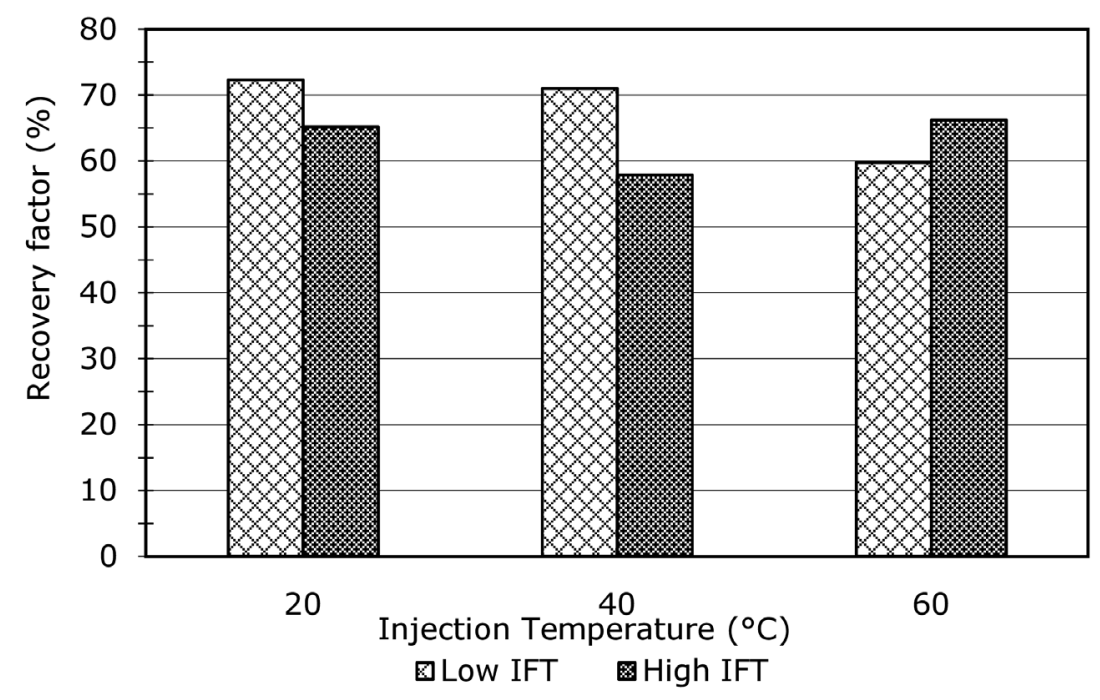

496

(a)

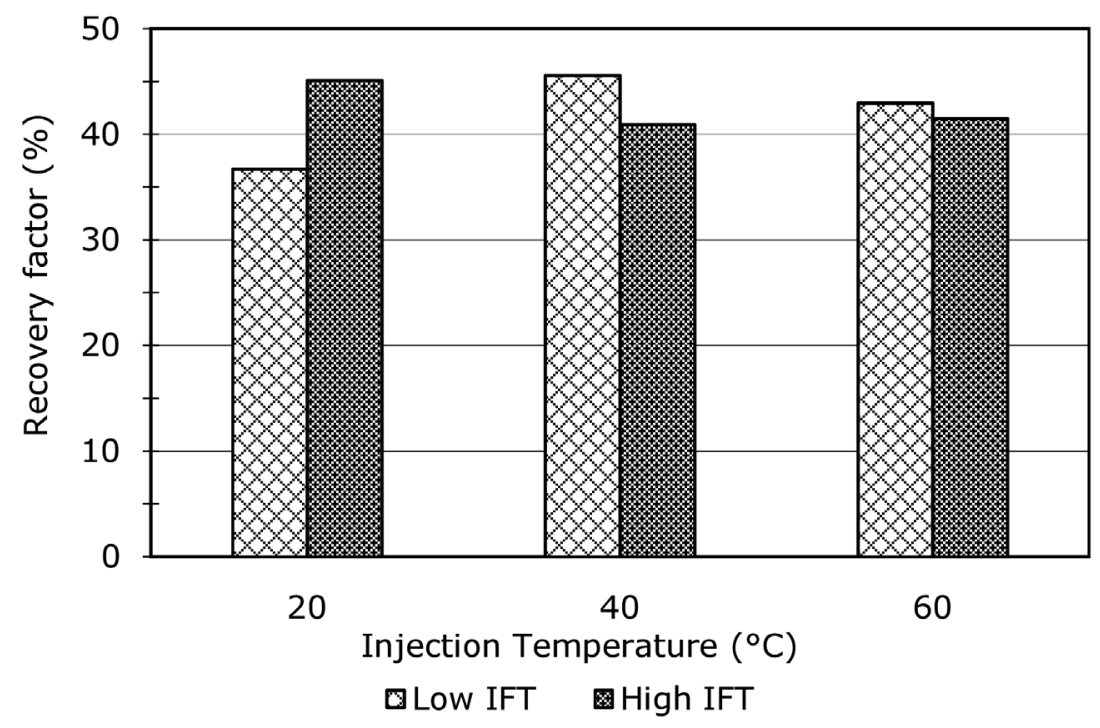




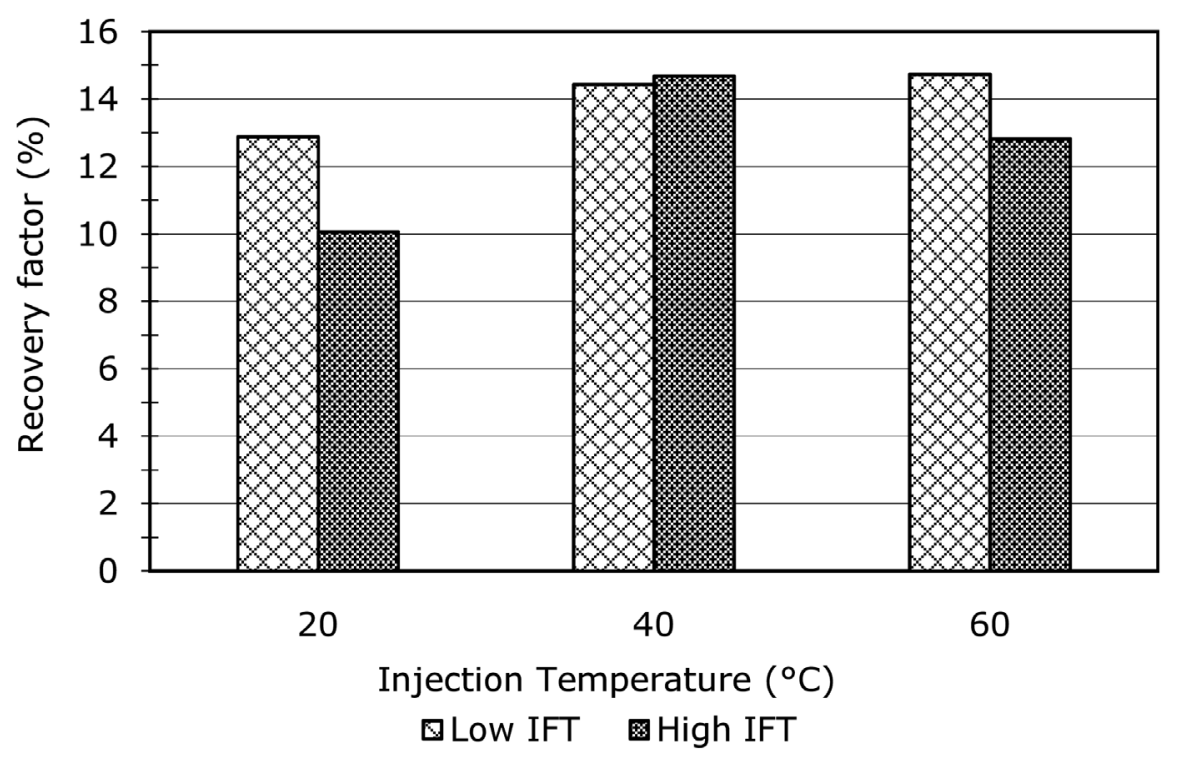

(c)

Figure 9: Combined effect of interfacial tension and temperature on recovery factor for oil-wet media (a) water-wet (b) intermediate-wet (c) oil-wet.

\section{Conclusion}

The results of the influence of wettability, temperature and interfacial tension (IFT) on water-flood oil recovery using an idealised pore-scale numerical model have been analysed. Reservoir conditions were used in all the cases with high and low IFT, waterwet, intermediate-wet and oil-wet state with an injection temperature of 20, 40 and 60 ${ }^{\circ} \mathrm{C}$. The main conclusions are:

i. Wettability alteration from oil-wet to water-wet condition can enhance the detachment of the oil phase from pore walls, but unable to improve the recovery sufficiently due to the capillary effect.

ii. The displacement efficiency in all the wettability conditions could be improved by a sufficient reduction in the interfacial tension between the fluid phases.

iii. Improved recovery could be achieved with a reduction in the interfacial tension and altering the wettability towards a water-wet state.

This study has demonstrated the effectiveness of using a computational fluid dynamics modelling approach to predict the influences of operating conditions on reservoir multiphase flow characteristics at pore-scale level. The use of the numerical approach will enhance the understanding of the requirements for the optimised oil recovery process.

At injection temperature of $20^{\circ} \mathrm{C}$ and high IFT of $0.045 \mathrm{~N} / \mathrm{m}$, a percentage recovery of about $10 \%$ was achieved while a reduced IFT to $0.025 \mathrm{~N} / \mathrm{m}$ gives a recovery of about $13 \%$. On the other hand, wettability reduction from the oil-wet to intermediate-wet states results in recovery factor of between $35-45 \%$ while a water-wet state results in recovery factor of between $60-75 \%$. This clearly shows that, though a low IFT is enough to resist the capillary effects, an improved oil recovery factor cannot be achieved due to the adherence of the oil to the walls caused by the wettability effects.

A comprehensive understanding of the pore-scale mechanisms that is controlling the macroscale displacement phenomenon as been investigated in this paper and will improve the predictive capability and design strategies for enhanced oil recovery practical applications and other fluid flow mechanism such as soil remediation, hydrology and sequestration of $\mathrm{CO}_{2}$ in deep saline aquifers. 
[1] E. C. Donaldson and M. Alam, Wettability, Elsevier, 2008.

[2] A. A. Jagar, K. Kamal, K. M. Abbas and H. M. Amir, "Recent advances in application of nanotechnology in chemical enhanced oil recovery: Effects of nanoparticles on wettability alteration, interfacial tension reduction, and flooding," Egyptian Journal of Petroleum, 2018.

[3] S. R. Harris, "Pore-scale investigation of wettability effects on two-phase flow in porous media," Doctor of philosophy thesis , University of Manchester, 2017.

[4] G. A. William, "Wettability Literature Survey - Part 1: Rock/Oil/Brine Interactions and the Effects of Core Handling on Wettabiiity," Journal of Petroleum Technology, no. 39, pp. 1125-1144, 1986.

[5] F. Olugbenga and M. Edo, "Wettability Effects on Capillary Pressure, Relative Permeability, and Irredcucible Saturation Using Porous Plate," Journal of Petroleum Engineering, vol. 2014, pp. 1-12, 2014.

[6] A. Chinedu, Y. D. Abhijit, K. Santanu and R. H. James, "The Effect of Wettability on Oil Recovery: A Review," Society opf Petroleum Engineers, no. 114496, 2008.

[7] J. M. Blunt, D. M. Jackson, M. Piri and H. P. Valvatne, "Detailed physics, predictive capabilities and macroscopic consequences for pore-network models of multiphase flow," Advances in Water Resources, no. 25, pp. 1069-1089, 2002.

[8] J. Buckley, Y. Liu and S. Monsterleet, "Mechanisms of Wetting Alteration by Crude Oils," SPE Journal, no. 37230, pp. 54-61, 1998.

[9] A. Wael, S. B. Jill, C. Andrew, E. John, H. Bernd, F. Edmund, G. Arne, H. Tarek, S. Nikita, S. Claude, H. Hassan, M. Bernard and Z. Murtaza, "Fundamentals of Wettability," Oilfield Review, pp. 44-61, 2007.

[10] G. A. William, "Wettability Literature Survey-Part 6: The Effects of Wettability on Waterflooding," Journal of Petroleum Technology, no. 39, pp. 1605-1622, 1987.

[11] K. Vitalii, "The Influence of Wettabillity on Oil Recovery," AGH Drilling, Oil, Gas, vol. 32, no. 3, pp. 493-502, 2015.

[12] Z. Peimao and A. Tor, "Wettability and oil recovery from carbonates: Effects of temperature and potential determining ions," Colloids and Surfaces A: Physicochem. Eng. Aspects, no. 279, pp. 179-187, 2006.

[13] W. Kallel, M. van Dijke, K. Sorbie, R. Wood, Z. Jiang and S. Harland, "Modelling the effect of wettability distributions on oil recovery from microporous carbonate reservoirs," Advances in Water Resources, no. 95, pp. 317-328, 2016.

[14] J. B. Martin, Z. Xiucai and Y. Jun, "Pore-scale modeling: Effects of wettability on waterflood oil recovery," Journal of Petroleum Science and Engineering, no. 71, pp. 169-178, 2010.

[15] P. Mohammadmoradi and K. Apostolos, "Pore Scale Investigation of Wettability Effect on Waterflood Performance," in SPE Annual Technical Conference and Exhibition, Dubai, 2016.

[16] L. Mingming, Shuzhong and Wang, "Pore-scale modeling of a water/oil two-phase flow in hot water flooding for enhanced oil recovery," RSC Advances, no. 5, pp. 85373-85382, 2015.

[17] Z. Jin and W. Dongsheng, "Pore-scale simulation of wettability and interfacial tension effects on flooding process for enhanced oil recovery," RSC Advances, no. 7, pp. 41391-41398, 2017.

[18] G. Tang and N. Morrow, "Salinity, temperature, oil composition and oil recovery by waterflooding," SPE Reservoir Engineering, vol. 12, pp. 269-276, 1997.

[19] J. Dangerfield and D. Brown, "The Ekofi sk Field: North Sea Oil and Gas Reservoirs," London, eds J. Kleepe et al., Graharm and Trotman, 1985, pp. 3-22. 
[20] J. M. Schembre, G. Tang and A. R. Kovscek, "Wettability Alteration and Oil Recovery by Water Imbibition at Elevated Temperatures," National Petroleum Technology Office, Tulsa, OK, 1998.

[21] M. Seán, "Chapter 7 - Clean water unit operation design: Physical processes," in An Applied Guide to Water and Effluent Treatment Plant Design, ButterworthHeinemann, 2018, pp. 69-100.

[22] Y. Ahmadi, M. Hassanbeygi and R. Kharrat, "The Effect of Temperature and Injection Rate during Water Flooding Using Carbonate Core Samples: An Experimental Approach," Iranian Journal of Oil \& Gas Science and Technology: Reservoir Engineering-EOR, vol. 5, no. 4, pp. 18-24, 2016.

[23] M. Amir, T. A. Mahmood and K.-P. Parissa, "The effect of temperature on the interfacial tension between crude oil and ethoxylated nonylphenols," Petroleum Science and Technology, vol. 34, no. 15, pp. 1315-1322, 2016.

[24] M. Sohel, S.-H. Tan and N.-T. Nguyen, "Temperature dependence of interfacial properties and viscosity of nanofluids for droplet-based microfluidics," Journal of Physics D: Applied Physics, vol. 41, no. 8, 2008.

[25] D. P. Konstantinos, A. M. Othonas and G. E. Ioannis, "Predictions of water/oil interfacial tension at elevated temperatures and pressures: A molecular dynamics simulation study with biomolecular force fields," vol. 476, pp. 30-38, 2017.

[26] J. U. Brackbill, D. B. Kothe and C. Zemach, "A Continuum Method for Modeling Surface Tension," Journal of Computational Physics, vol. 100, pp. 335-354, 1992.

[27] F. ANSYS, "Overview and Limitations of the VOF Model," Fluent Inc., 2006.

[28] "Oilfield Glossary: Schlumberger," Schlumberger Limited, [Online]. Available: https://www.glossary.oilfield.slb.com/en/Terms/c/capillary_number.aspx. [Accessed 18 January 2019].

[29] A. Carcoana, Applied Enhanced Oil Recovery, Prentice Hall, 1992.

[30] S. Thomas, "Enhanced Oil Recovery - An Overview," Oil \& Gas Science and Technology, vol. 63, no. 1, pp. 9-19, 2007. 